\title{
Oncogenic Addiction to ERBB2 Signaling Predicts Response to Trastuzumab in Urothelial Cancer
}

\author{
Michael Karass, MDa; Rohan Bareja, MSc $c^{\mathrm{b}, c}$; Ethan Shelkey ${ }^{\mathrm{d}}$; Panagiotis J. Vlachostergios, MD, PhDe; \\ Brian D. Robinson, MDf,g; Francesca Khani, MD;; Juan Miguel Mosquera, MD, MSc,g; Douglas S. Scherr, MD ; \\ Andrea Sboner, MS, PhD b,c,g; Scott T. Tagawa, MD, MS ${ }^{e, g, h, i}$; Ana M. Molina, MD ${ }^{e, g, h, i} ;$ Olivier Elemento, PhD ${ }^{b, c, g, i ;}$ \\ David M. Nanus, MD ${ }^{e, g, h, i}$; and Bishoy M. Faltas, MDe,g,i
}

\section{ABSTRACT}

Urothelial carcinoma (UC) is a common and frequently lethal cancer. Despite the presence of genomic alterations creating dependency on particular signaling pathways, the use of targeted therapies in advanced and metastatic UC has been limited. We performed an integrated analysis of whole-exome and RNA sequencing of primary and metastatic tumors in a patient with platinum-resistant UC. We found a strikingly high ERBB2 mRNA expression and enrichment of downstream oncogenic ERBB2 signaling in this patient's tumors compared with tumors from an unselected group of patients with UC ( $N=17)$. This patient had an exceptional sustained response to trastuzumab. Our findings show that oncogenic addiction to ERBB2 signaling potentially predicts response to ERBB2-directed therapy of UC.

J Natl Compr Canc Netw 2019;17(3):194-200 doi: 10.6004/jnccn.2018.7264

a'Division of Internal Medicine, New York Medical College, Westchester Medical

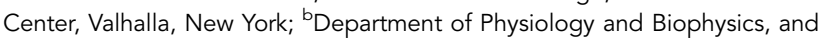
Institute for Computational Biomedicine, Weill Cornell Medicine, New York, New York; 'Wake Forest Institute for Regenerative Medicine, Wake Forest School of Medicine, Winston-Salem, North Carolina; and ' ${ }^{\mathrm{D}}$ epartment of Medicine, Division of Hematology and Medical Oncology, fDepartment of Pathology and Laboratory Medicine, 9Englander Institute for Precision Medicine, hepartment of Urology, and 'Sandra and Edward Meyer Cancer Center, Weill Cornell Medicine, New York, New York.
Exceptional responders are patients who experience deep and durable responses to treatments that are not effective for most of the population with the same malignancy. ${ }^{1}$ Studying the molecular basis for these extreme phenotypes provides insight into the molecular determinants of response to targeted therapy. ${ }^{2}$ To dissect the determinants of sensitivity to ERBB2-directed therapy in a patient with metastatic platinum-resistant urothelial cancer (PRUC) who achieved an exceptional response to trastuzumab, we performed whole-exome sequencing (WES) and RNA sequencing (RNA-seq) of the patient's primary and metastatic PRUC tumors. We discovered evidence of oncogenic addiction to ERBB2 signaling in this patient's tumors.

\section{Methods}

\section{Patient Details}

Patients consented to participate in the precision medicine protocol at Weill Cornell Medicine (WCM), and the study was approved by the WCM/New York-Presbyterian Institutional Review Board (protocol 1305013903). Samples from the patient's cystectomy tumor and metastases were prospectively collected. Somatic DNA from tumor samples and germline DNA from peripheral blood mononuclear cells were submitted for WES as previously described. ${ }^{3}$ RNA was extracted from tumor samples and submitted for RNA-seq.

\section{Whole-Exome Sequencing}

DNA extractions from patients' tumors were performed using the Maxwell 16 Tissue DNA Purification Kit (Promega). Whole-exome capture libraries were constructed after sample shearing, end repair, and phosphorylation and ligation to barcoded sequencing adaptors. Ligated DNA was size-selected for lengths between 200 and $350 \mathrm{bp}$ and subjected to HaloPlex Exome Target Enrichment System sequencing (Agilent Technologies). Sequencing was performed using the high-throughput HiSeq 2500 system $(2 \times 100 \mathrm{bp}$; Illumina). Reads were aligned 
to GRCh37/hg19 reference using the Burrows-Wheeler Aligner and processed according to the Institute for Precision Medicine IPM-Exome-pipeline, version 0.9. ${ }^{3}$

\section{RNA-Seq Analysis of WCM Samples}

We used STAR_2.4.0f1 for sequence alignment against the human genome build hg19, downloaded via the UCSC Genome Browser, and Sequence Alignment/Map SAMtools version 0.1.19 for sorting and indexing reads, Cufflinks 2.0.2 for obtaining the expression values (fragments per kilobase of exon per million fragments mapped [FPKM]), and UCSC RefGene GTF (gene transfer format) file for annotation.

\section{Gene Set Enrichment Analysis}

GSEAPreranked was used for gene set enrichment analysis (GSEA). $\log _{2}$ fold change of the WCM137-1 versus the mean of other WCM bladder cases was calculated for all genes, which became the input for GSEA. Similarly, we calculated the fold change for WCM137-2. Gene sets used were oncogenic signatures and 90 curated gene sets of druggable cancer genes. Significant pathways with $P<.05$ and false discovery rates $<0.25$ are reported.

\section{Outlier Analysis}

We used STAR_2.4.0f1 for sequence alignment against the human genome build hg19, downloaded via the UCSC Genome Browser, and SAMtools version 0.1.19 for sorting and indexing reads, Cufflinks 2.0.2 for obtaining the expression values (FPKM), and GENCODE version 19 GTF files for annotation. ${ }^{4-7}$ The gene counts from the HTSeq count and DESeq2 Bioconductor package were used to identify differentially expressed genes. ${ }^{8,9}$ The hypergeometric test and GSEA ${ }^{10}$ were used to identify enriched signatures using the different pathway collections in the MSigDB database (Molecular Signatures Database). ${ }^{11}$

Z-scores were calculated across the WCM bladder cohort for 74 druggable genes. For each sample, the quantiles were calculated to compute the lower and upper bounds to define an outlier. We selected genes with z-scores $>2.5$ and FPKM $>100$ as outlier genes.

\section{Results}

Clinical Course and Exceptional Response

A 76-year-old woman initially presented with hematuria. Cystoscopy and transurethral resection of bladder tumor (TURBT) revealed high-grade $\mathrm{T} 1$ urothelial carcinoma (UC) (Figure 1A). A restaging TURBT performed 6 weeks later revealed residual malignancy with superficial invasion into the lamina propria. The patient was treated with intravesical bacillus Calmette-Guérin (BCG) and experienced remission for 25 months until she developed a local recurrence. She was retreated with a second course of intravesical BCG but 5 months later started experiencing urinary obstruction and was found to have a mass at the tip of the urethra. A biopsy of this mass revealed high-grade UC with extensive invasion of the surrounding connective tissues. FDG-PET/ CT revealed bilateral inguinal lymphadenopathy. A biopsy of the inguinal lymph nodes revealed metastatic UC.

The patient was referred for systemic therapy and was enrolled in the CALGB 90601 randomized phase III trial comparing gemcitabine + cisplatin (GC) and bevacizumab with GC and placebo in patients with advanced UC. She received 6 cycles of treatment (GC \pm bevacizumab). Restaging CT scans showed a partial response in the pelvic lymph nodes along with persistence of high-grade papillary muscle-invasive UCB on repeat TURBT. Because her disease remained confined to the pelvis, she underwent a robot-assisted radical cystectomy with ileal conduit urinary diversion and left inguinal lymph node excision (pathologic stage ypT2N2M0). At 5 months after surgery, she developed systemic disease progression presenting with new liver metastases (Figure 1B). To identify potentially druggable genetic alterations, we enrolled her in our Institute for Precision Medicine protocol study and performed an in-depth integrated analysis of WES and RNA-seq from her primary and metastatic PRUC tumors, which showed outlier ERBB2 mRNA expression (measured in FPKM) (Figure 2A). In fact, ERBB2 FPKMs from the primary and metastatic tumors were ranked 62nd and 53rd, respectively, of 21,000 genes when ranked by expression level (Figure 2A). Allelespecific copy number analysis of the ERBB2 locus on chromosome 17 showed strikingly high levels of focal $E R B B 2$ amplification (Figure 2B, C) with copy number values of 14 and 20 in the primary and metastatic tumors, respectively (Figure 2C). These findings were validated by fluorescence in situ hybridization (FISH) (Figure 2D). The outlier somatic ERBB2 copy number and mRNA expression translated into overexpression of the ERBB2 protein on immunohistochemical (IHC) analysis (Figure 2E). No activating somatic mutations in ERBB2 were identified.

Based on these results, the patient was started on trastuzumab $(6 \mathrm{mg} / \mathrm{kg})$ in combination with docetaxel $\left(70 \mathrm{mg} / \mathrm{m}^{2}\right)$. She received 4 cycles of this regimen, which were well-tolerated, and experienced substantial clinical improvement. Surprisingly, restaging CT scans showed a complete response with total disappearance of all metastatic lesions (Figure 1B). She continued on maintenance trastuzumab every 3 weeks. Four years later, she continues to be in complete remission with no evidence of disease. 
A

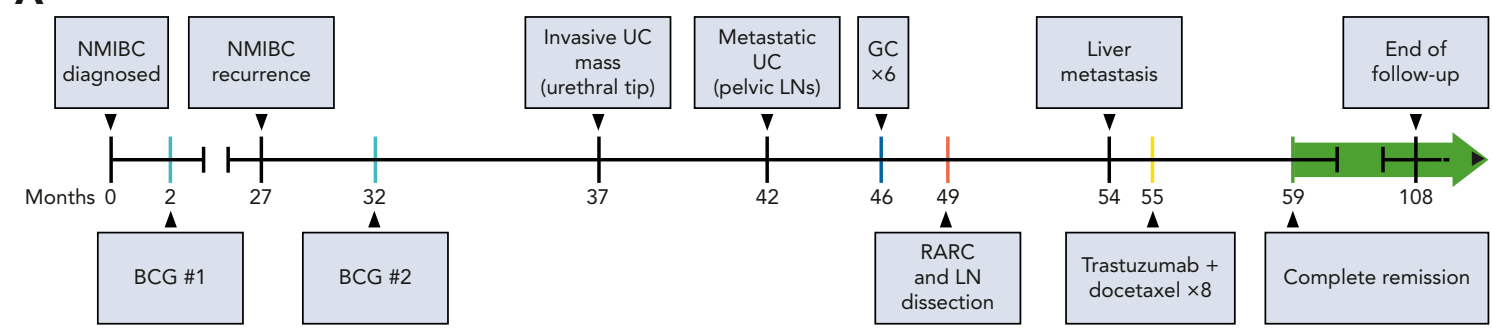

B
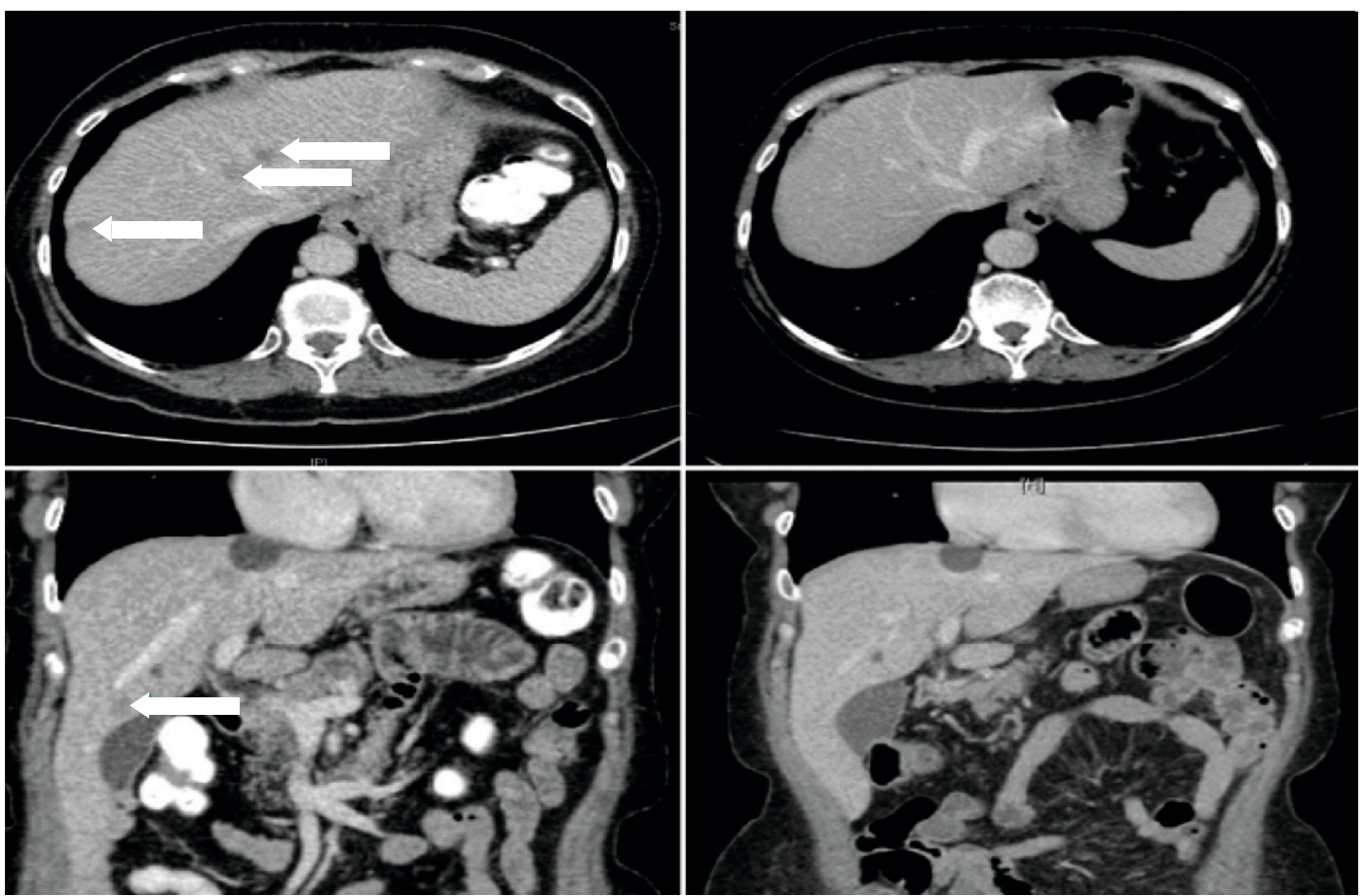

Figure 1. Patient's clinical course. (A) Clinical events in chronologic order, including the diagnosis of noninvasive, invasive, and metastatic disease; local and systemic treatments at each stage; outcomes; and last follow-up (time scale, months). (B) Axial and coronal CT images showing liver metastases (left panels, arrows) and complete response after docetaxel + trastuzumab (right panels).

Abbreviations: BCG, bacillus Calmette-Guérin; GC, gemcitabine/cisplatin; LNs, lymph nodes; NMIBC, non-muscle-invasive bladder cancer; RARC, robot-assisted radical cystectomy; UC, urothelial cancer.

We reasoned that the exceptional response phenotype observed in this patient's PRUC resulted from targeting the tumor's addiction to ERBB2 signaling. We hypothesized that oncogenic addiction to ERBB2 requires outlier overexpression of genes specifically involved in signal transduction through the ERBB2 receptor tyrosine kinase pathway. To test this hypothesis, we performed RNA-seq analysis of an unselected cohort of patients with advanced UC $(n=17)$ from our institution and compared ERBB2 mRNA expression in their tumors with that from our exceptional responder's tumors. ERBB2 mRNA expression in both the primary and metastatic samples from our patient was significantly higher compared with other patients in the cohort $(P=4.229 \mathrm{e}-08)$ (Figure 3A). Analysis of outlier mRNA expression ranked the exceptional responder's ERBB2 expression in the 95th percentile of all transcripts compared with tumors from other unselected patients in the cohort (Figure 3B).

To determine whether this ERBB2 outlier mRNA expression level translates into actual activation of ERBB2 downstream signaling, we performed GSEA. We used the gene expression data from normal bladder tissue in the Genotype-Tissue Expression (GTEx) project database as a normal control. ${ }^{12}$ GSEA revealed significant enrichment in the expression of ERBB2-target genes (ERBB2 signature) in tumors from our exceptional responder compared with normal bladder tissue from GTEx and UBC tumors in our unselected cohort (Figure 3C). These results strongly suggest that our exceptional responder's tumors were specifically dependent on the 
A
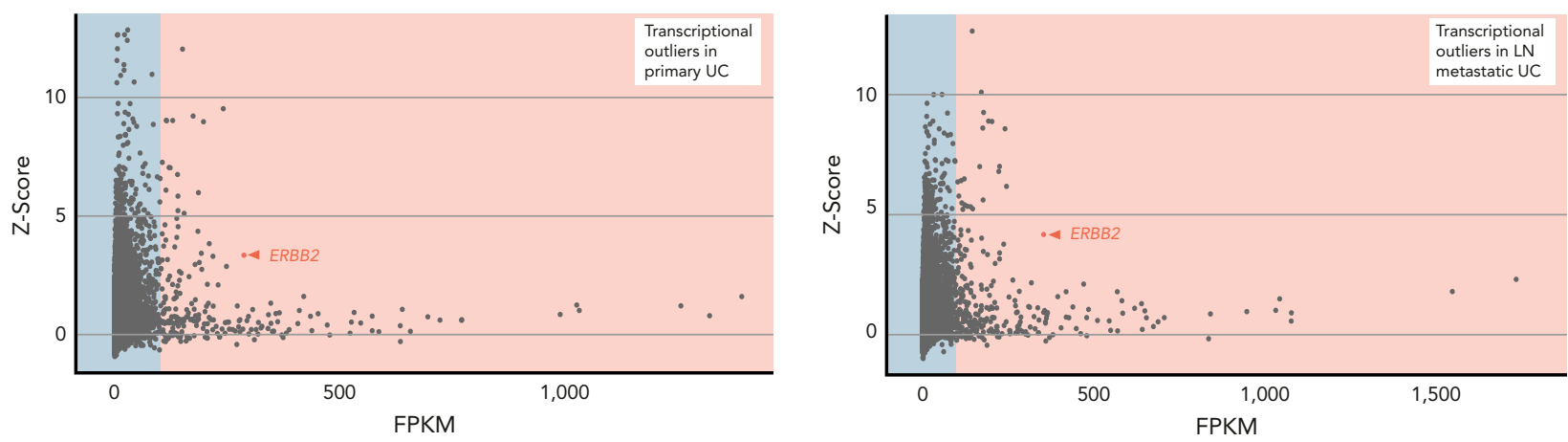

B

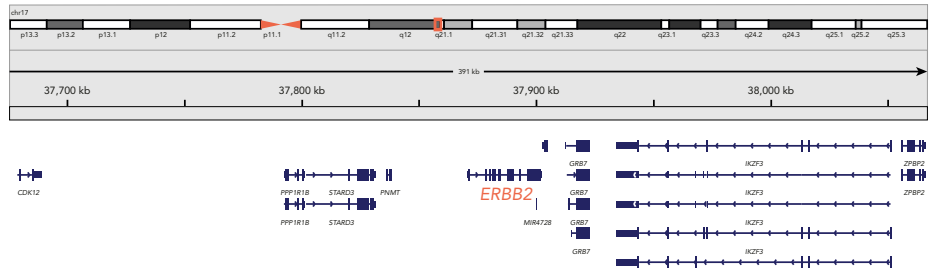

D

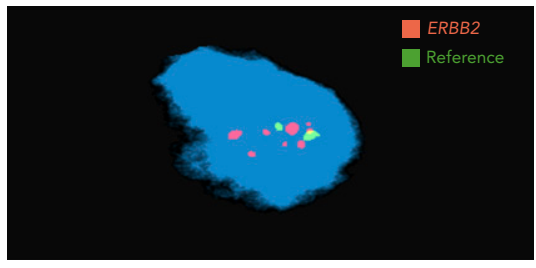

C
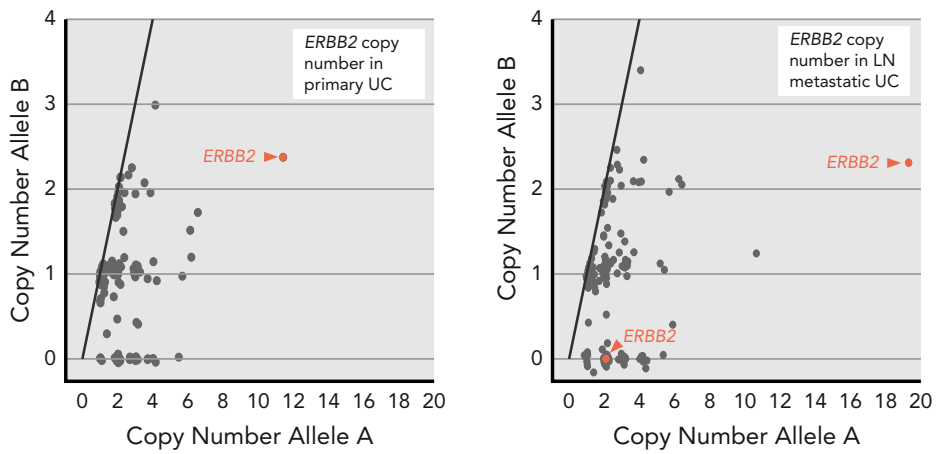

E

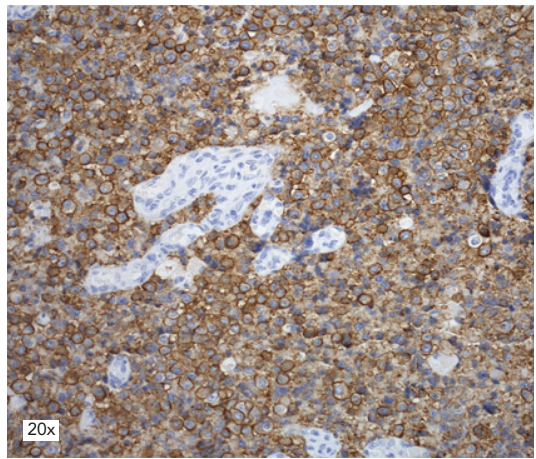

Figure 2. ERBB2 overexpression in the patient's tumors. (A) ERBB2 mRNA outlier expression in primary (left panel) and metastatic tumors (right panel). (B) Location of the ERBB2 locus on chromosome 17. (C) Allele-specific copy number analysis of the ERBB2 locus showing strikingly high levels of focal ERBB2 amplification in the primary and metastatic tumors. (D) Fluorescence in situ hybridization confirms the presence of several copies of ERBB2 (red) per nucleus compared with 2 reference copies of chromosome 17 (green) (original magnification x600). (E) Diffuse ERBB2 staining by immunohistochemistry (original magnification $\times 200$ ).

Abbreviations: FPKM, fragments per kilobase of exon per million fragments mapped; LN, lymph node; UC, urothelial cancer.

outlier ERBB2 expression and the ensuing constitutive activation of a network of ERBB2 target genes. This resulted in a phenotype that was rapidly progressive but exceptionally sensitive to ERBB2-directed therapy.

\section{Discussion}

The ERBB/HER signaling pathway is a receptor-ligand system that consists of 4 receptors and at least 11 ligands. ${ }^{13}$ ERBB2 is a member of the ERBB receptor family that acts as a coreceptor with ERBB1, ERBB3, and ERBB4. The humanized monoclonal antibody trastuzumab is directed against ERBB2 and approved for the treatment of several ERBB2-overexpressing cancers, including breast and gastric. ${ }^{14,15}$ Molecular alterations in ERBB2 are frequent in invasive UC, with a reported prevalence of $9.2 \%$ to $80 \%$ for protein overexpression and up to $32 \%$ for copy number amplification. ${ }^{16,17}$ ERBB2 overexpression in UC is associated with increased metastasis and recurrence. ${ }^{18}$

Several clinical trials of ERBB2-directed therapies in UC failed to meet their primary end points ${ }^{19-21}$ but demonstrated activity in a subset of patients with tumors overexpressing ERBB2, including some patients with durable responses..$^{14,22}$ In a single-arm phase II study of 59 patients with locally advanced or metastatic UC, 
A

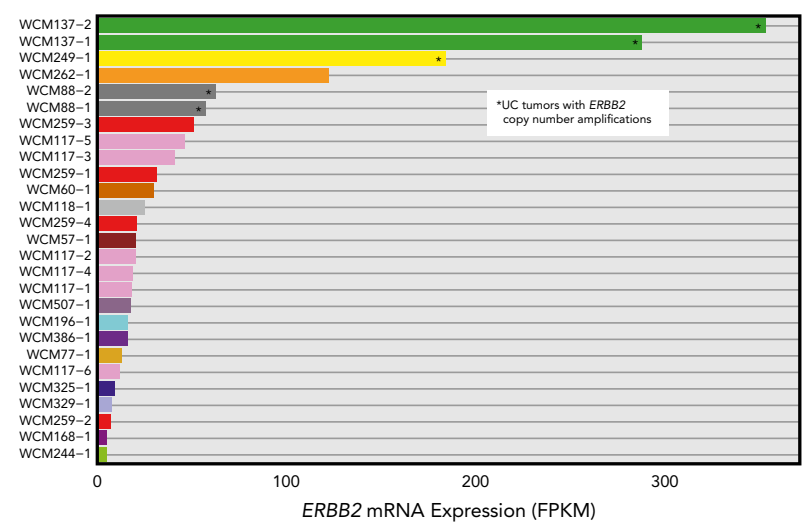

C
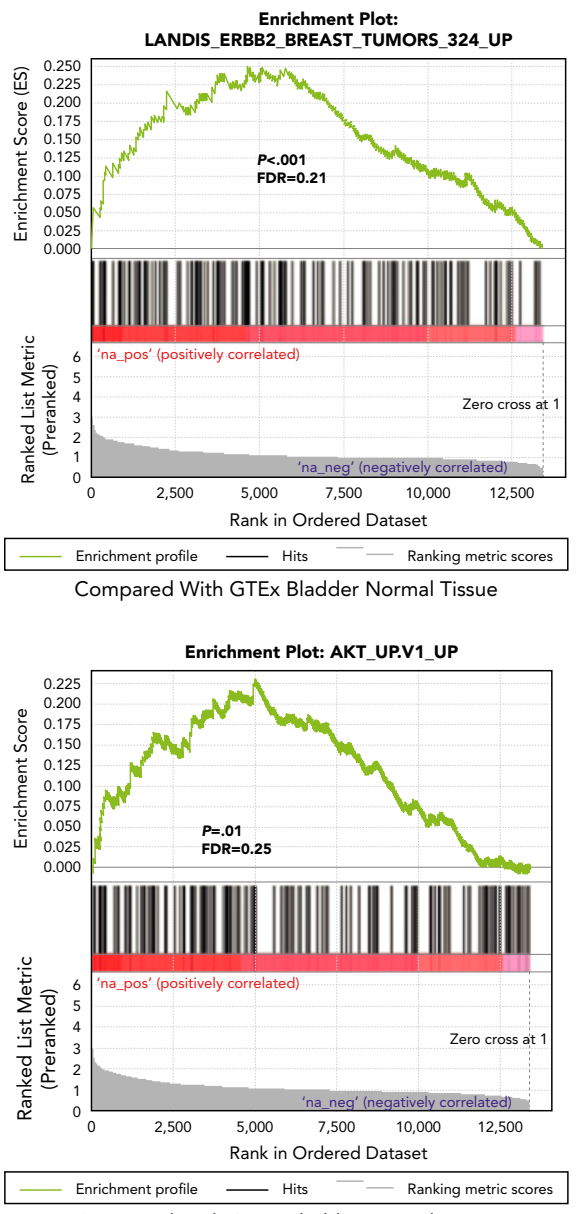

B

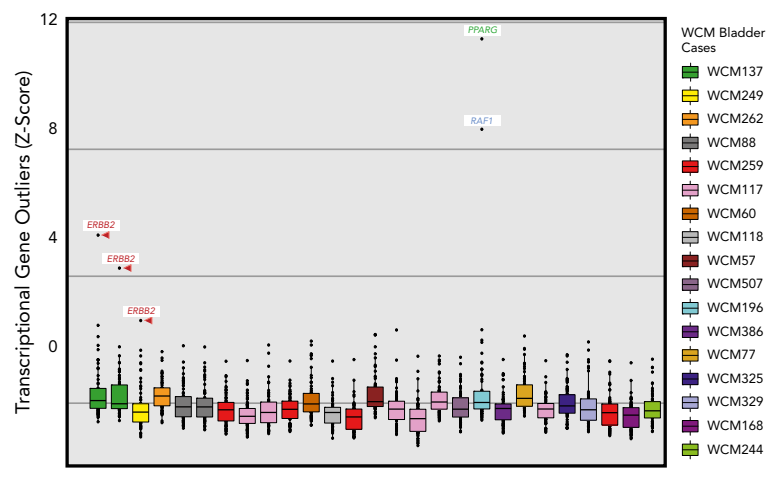

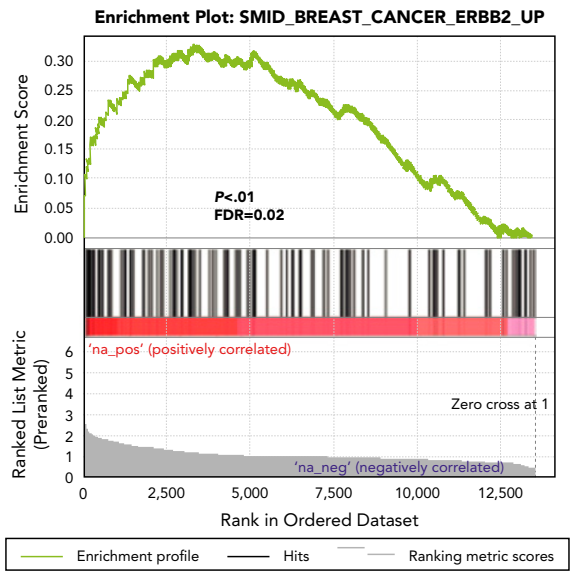

Compared With GTEx Bladder Normal Tissue

Enrichment Plot:

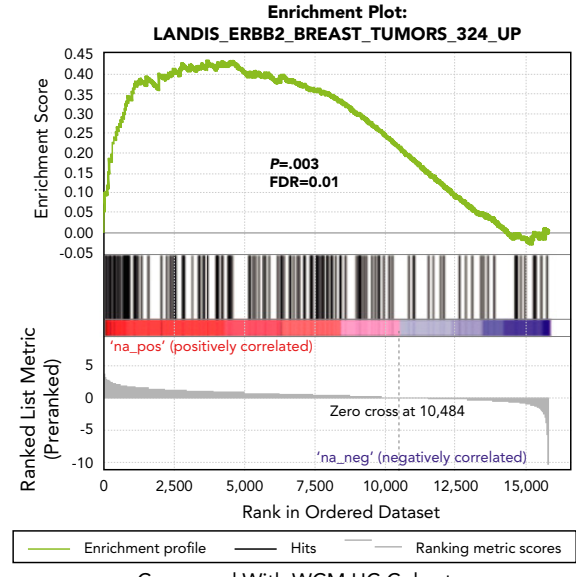

Figure 3. ERBB2 mRNA expression was significantly higher in tumors from the exceptional responder than in unselected patients with UC and normal bladder tissue. (A) Primary and metastatic samples from the exceptionally responding patient have significantly higher ERBB2 mRNA levels (WCM137, top 2 green bars) than those from patients with UC from the unselected WCM cohort $(P=4.229 \mathrm{e}-08)$. (B) Outlier analysis of ERBB2 mRNA expression confirms ERBB2 as an outlier gene in the exceptional responder's primary and metastatic tumor samples (ordered from left to right) compared with tumors from other patients in the unselected WCM UC cohort. (C) ERBB2 target gene transcripts are enriched in gene set enrichment analysis of tumors from the exceptional responder compared with normal bladder tissue from GTEx and tumors from the unselected WCM UC cohort.

Abbreviations: FDR, false discovery rate; FPKM, fragments per kilobase of exon per million fragments mapped; GTEx, Genotype-Tissue Expression; UC, urothelial cancer; WCM, Weill Cornell Medicine. 
lapatinib, a dual-receptor tyrosine kinase inhibitor of ERBB1 and ERBB2, was used as second-line treatment. ${ }^{19}$ Although the response rate $(1.7 \%)$, time to progression (8.6 weeks), and overall survival (17.9 weeks) did not meet the primary end point, patients with overexpression of ERBB1 or ERBB2 had an improved median overall survival of 30.3 versus 10.6 weeks in those with low or negative expression $(P=.0001)$. In another single-arm phase II trial in which treatment-naïve patients with advanced UC were treated with trastuzumab, paclitaxel, carboplatin, and gemcitabine, ${ }^{14}$ ERBB2 overexpression by IHC, gene amplification in the tumor by FISH, or presence in serum for extracellular ERBB2 domain testing by quantitative enzyme-linked immunosorbent assay was detected in $52 \%$ of patients. In this trial, $70 \%$ of patients with ERBB2-overexpressing UC tumors experienced a clinical response to the chemotherapy combination; median time to progression and median survival were 9.3 and 14.1 months, respectively. Although paclitaxel could have had a role in the exceptional response we observed in our patient, the persistence of the complete response after discontinuation of paclitaxel while receiving maintenance trastuzumab is consistent with possible oncogenic addiction to ERBB2 signaling, as suggested by the presence of ERBB2 transcriptional signature.

In another phase II trial, 61 patients with advanced UC and overexpression of ERBB2 by IHC and confirmed by FISH were randomized to receive GC or gemcitabine + carboplatin without (arm A) or with (arm B) trastuzumab. ${ }^{20}$ The trial did not demonstrate significant differences between the arms. Recently, a phase II trial by Choudhury et $\mathrm{al}^{21}$ examined the 3-month progression-free survival (PFS3) in patients with metastatic PRUC treated with afatinib, $40 \mathrm{mg} / \mathrm{d}$ continuously until progression or intolerance. Tumor analysis for alterations in EGFR, ERBB2, ERBB3, and ERBB4 was performed using next-generation sequencing. Of 23 patients, $5(21.7 \%)$ achieved PFS3, but because the study did not meet the prespecified end point of $\geq 7$ patients reaching PFS3, the study did not advance to the second stage of enrollment. For the entire cohort, the median PFS was 1.4 months, the objective response rate was $8.6 \%$, and the median overall survival for all patients was 5.3 months. The 6 patients with $E R B B 2 / 3$ alterations had a median time to progression of 6.6 months compared with 1.4 months in patients without alterations $(P<.001)$. In this trial, $83 \%$ of patients with ERBB2 copy number amplification and/or ERBB3 somatic mutations achieved PFS3, whereas $0 \%$ of patients without alterations reached PFS3. The patient with the longest PFS had the highest somatic copy number of ERBB2 (copy number, $>50$ ) and was the only patient with both ERBB2 and ERBB3 alterations. ${ }^{21}$ This finding is consistent with our observation that outlier ERBB2 mRNA and copy number levels are associated with response to ERBB2-targeted therapy.
Collectively, the results of these trials highlight the challenges in using ERBB2 status as a biomarker for trials of ERBB2-targeted therapies. It is important to recognize that $E R B B 2$ copy number amplification does not linearly translate into protein overexpression by IHC.,21 Furthermore, the relevance of $E R B B 2$ as a tumor driver may vary even within ERBB2-amplified cases. ${ }^{23}$ The result of the Choudhury et $\mathrm{al}^{21}$ study in UC showed that ERBB2 amplification by quantitative PCR or FISH copy number was more predictive of response to ERBB2-directed therapy with afatinib in patients with UC compared with protein overexpression detected by $\mathrm{IHC}^{10} ; 75 \%$ of patients with amplification reached PFS3 compared with only $25 \%$ with $2+$ or $3+$ IHC staining. ${ }^{21}$ IHC testing to evaluate HER2 status is notoriously inaccurate compared with FISH. ${ }^{10} \mathrm{~A}$ meta-analysis evaluating the concordance between IHC and FISH for detecting ERBB2 in breast cancer reported rates of $36 \%$ for IHC score $2+$ and $91 \%$ for IHC score $3+.^{24}$ These findings highlight the challenges of using a single biomarker test to predict response to ERBB2directed therapy.

\section{Conclusions}

Our report describes an integrated analysis of WES, RNAseq, IHC, and FISH suggesting that oncogenic addiction to ERBB2 is associated with exceptional clinical response to trastuzumab. Because most trials testing ERBB2directed therapies have relied on assigning ERBB2 status by IHC, it is possible that the overall negative results of these trials might reflect a failure of the biomarker methodology to predict oncogenic addiction to ERBB2 rather than a failure of the tested ERBB2-targeted therapies. A combinatorial biomarker strategy that integrates genomic and RNA-seq, FISH, and protein quantification might predict oncogenic addiction to a specific signaling pathway. Future testing of ERBB2directed treatments in a subset of patients with UC tumors with oncogenic addiction to ERBB2 is warranted.

Submitted July 30, 2018; accepted for publication December 18, 2018.

Author contributions: Study concept, design, and supervision: Faltas. Experiment performance and analysis: Bareja, Robinson, Khani, Mosquera, Sboner, Elemento, Faltas. Sequencing data analysis: Bareja, Sboner, Elemento, Faltas. Immunohistochemical staining: Robinson, Khani, Mosquera. Data interpretation: Karass, Shelkey, Vlachostergios, Faltas. Manuscript preparation: Karass, Shelkey, Vlachostergios, Faltas. Critical revision: Karass, Robinson, Khani Mosquera, Scherr, Sboner, Tagawa, Molina, Elemento, Nanus, and Faltas.

Disclosures: Dr. Tagawa has disclosed that he has received grant/research support from Genentech. Dr. Faltas has disclosed that he has received grant/ research support from Eli Lilly and Company. The remaining authors have not received any financial consideration from any person or organization to support the preparation, analysis, results, or discussion of this article.

Funding: Dr. Faltas has received funding from the Department of Defense CDMRP Career Development Award (grant CA160212).

Correspondence: Bishoy M. Faltas, MD, Hematology and Medical Oncology, Weill Cornell Medicine, 520 East 70th Street, New York, NY 10021. Email: bmf9003@med.cornell.edu 


\section{References}

1. Mullard A. Learning from exceptional drug responders. Nat Rev Drug Discov 2014;13:401-402.

2. Chang DK, Grimmond SM, Evans TR, et al. Mining the genomes of exceptional responders. Nat Rev Cancer 2014;14:291-292.

3. Rennert H, Eng K, Zhang $T$, et al. Development and validation of a wholeexome sequencing test for simultaneous detection of point mutations, indels and copy-number alterations for precision cancer care. NPJ Genom Med 2016;1:pii: 16019

4. Trapnell C, Roberts A, Goff L, et al. Differential gene and transcript expression analysis of RNA-seq experiments with TopHat and Cufflinks [Erratum in Nat Protoc 2014;9:2513]. Nat Protoc 2012;7:562-578.

5. Li H, Handsaker B, Wysoker A, et al. The Sequence Alignment/Map format and SAMtools. Bioinformatics 2009;25:2078-2079.

6. Dobin A, Davis CA, Schlesinger F, et al. STAR: ultrafast universal RNA-seq aligner. Bioinformatics 2013;29:15-21.

7. Derrien T, Johnson R, Bussotti G, et al. The GENCODE v7 catalog of human long noncoding RNAs: analysis of their gene structure, evolution and expression. Genome Res 2012;22:1775-1789.

8. Anders S, Pyl PT, Huber W. HTSeq-a Python framework to work with high-throughput sequencing data. Bioinformatics 2015;31:166-169.

9. Love MI, Huber W, Anders S. Moderated estimation of fold change and dispersion for RNA-seq data with DESeq2. Genome Biol 2014; 15:550.

10. Subramanian A, Tamayo P, Mootha VK, et al. Gene set enrichment analysis: a knowledge-based approach for interpreting genomewide expression profiles. Proc Natl Acad Sci USA 2005;102 15545-15550.

11. Liberzon A, Subramanian A, Pinchback R, et al. Molecular signatures database (MSigDB) 3.0. Bioinformatics 2011;27:1739-1740.

12. GTEx Consortium. The Genotype-Tissue Expression (GTEx) project. Nat Genet 2013;45:580-585.

13. Yarden Y, Sliwkowski MX. Untangling the ErbB signalling network. Nat Rev Mol Cell Biol 2001;2:127-137.

14. Hussain MHA, MacVicar GR, Petrylak DP, et al. Trastuzumab, paclitaxel carboplatin, and gemcitabine in advanced human epidermal growth factor receptor-2/neu-positive urothelial carcinoma: results of a multicenter phase II National Cancer Institute trial. J Clin Oncol 2007;25: 2218-2224.

15. Gomez-Martin C, Plaza JC, Pazo-Cid R, et al. Level of HER2 gene amplification predicts response and overall survival in HER2-positive advanced gastric cancer treated with trastuzumab. J Clin Oncol 2013;31:4445-4452.

16. Miyamoto $\mathrm{H}$, Kubota $\mathrm{Y}$, Noguchi S, et al. C-ERBB-2 gene amplification as a prognostic marker in human bladder cancer. Urology 2000;55:679-683.

17. Laé M, Couturier J, Oudard $\mathrm{S}$, et al. Assessing HER2 gene amplification as a potential target for therapy in invasive urothelial bladder cancer with a standardized methodology: results in 1005 patients. Ann Oncol 2010;21:815-819.

18. Fleischmann A, Rotzer $D$, Seiler R, et al. Her2 amplification is significantly more frequent in lymph node metastases from urothelial bladder cancer than in the primary tumours. Eur Urol 2011;60:350-357.

19. Wülfing $C$, Machiels JP, Richel DJ, et al. A single-arm, multicenter, openlabel phase 2 study of lapatinib as the second-line treatment of patients with locally advanced or metastatic transitional cell carcinoma. Cancer 2009:115:2881-2890.

20. Oudard S, Culine S, Vano Y, et al. Multicentre randomised phase II trial of gemcitabine + platinum, with or without trastuzumab, in advanced or metastatic urothelial carcinoma overexpressing Her2. Eur J Cancer 2015; 51:45-54.

21. Choudhury NJ, Campanile A, Antic T, et al. Afatinib activity in platinumrefractory metastatic urothelial carcinoma in patients with ERBB alterations. J Clin Oncol 2016;34:2165-2171.

22. Coombs LM, Pigott DA, Sweeney E, et al. Amplification and overexpression of c-erbB-2 in transitional cell carcinoma of the urinary bladder. Br J Cancer 1991;63:601-608

23. Kiss B, Wyatt AW, Douglas J, et al. Her2 alterations in muscle-invasive bladder cancer: patient selection beyond protein expression for targeted therapy. Sci Rep 2017;7:42713.

24. Bahreini F, Soltanian AR, Mehdipour P. A meta-analysis on concordance between immunohistochemistry $(\mathrm{IHC})$ and fluorescence in situ hybridization (FISH) to detect HER2 gene overexpression in breast cancer. Breast Cancer 2015;22:615-625

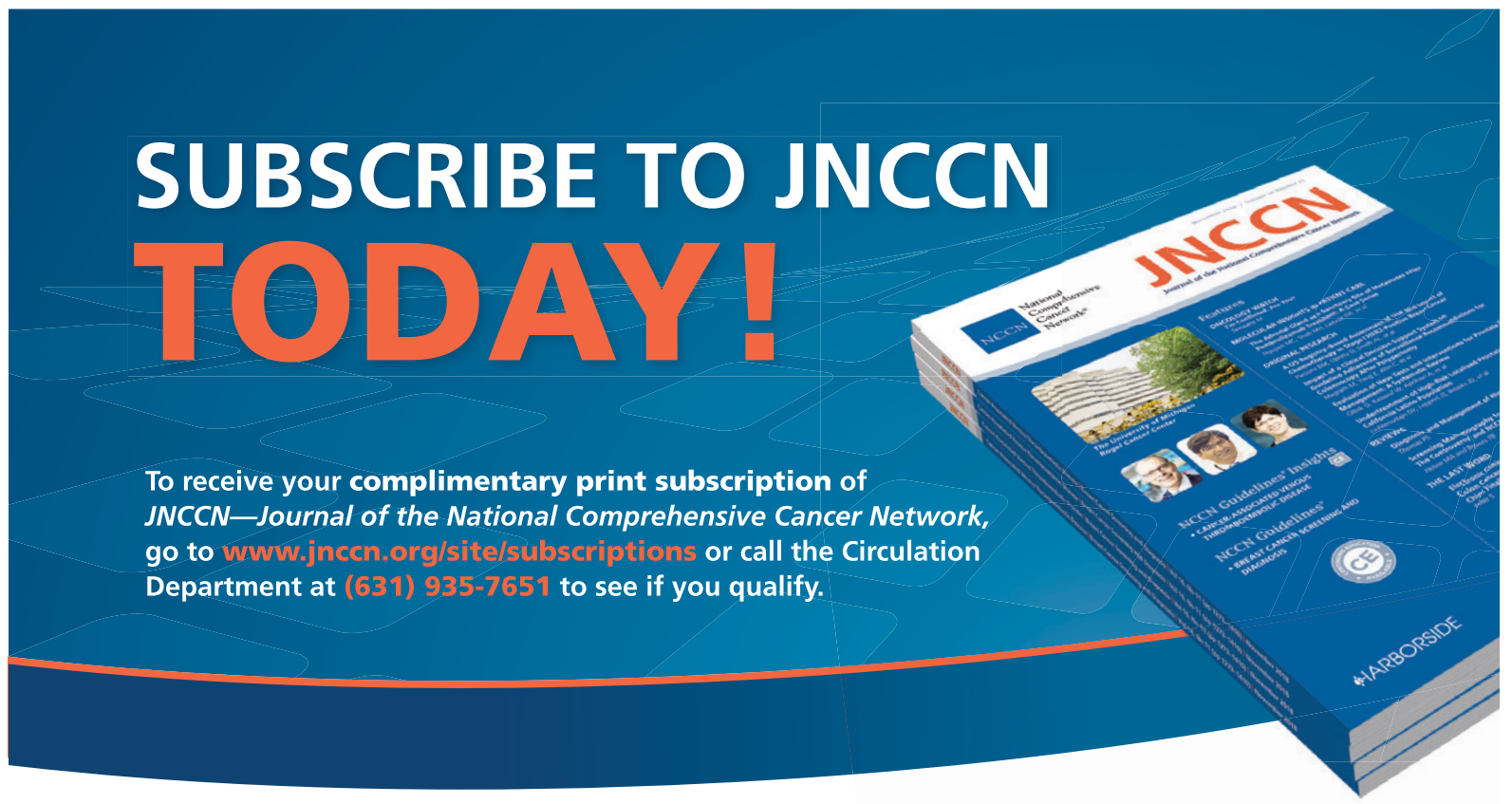

Your print subscription also grants you access to JNCCN.org! Access archived issues from 2003 to the present, bonus online reviews, and additional information on continuing education activities.

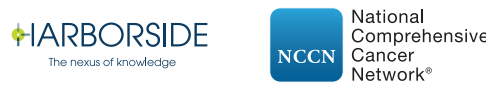

